\title{
How commonly is upper gastrointestinal cancer missed at endoscopy? A meta-analysis
}

Authors

Institutions
Shyam Menon ${ }^{1}$, Nigel Trudgill ${ }^{2}$

${ }^{1}$ Department of Gastroenterology, The Royal Wolverhampton Hospitals NHS Trust, New Cross Hospital, Wolverhampton, United Kingdom

${ }^{2}$ Department of Gastroenterology, Sandwell General Hospital, West Bromwich, United Kingdom submitted 8. October 2013 accepted after revision 17. February 2014

\section{Bibliography}

DOI http://dx.doi.org/

10.1055/s-0034-1365524

Published online: 7.5.2014

Endoscopy International Open 2014; 02: E46-E50

(c) Georg Thieme Verlag KG

Stuttgart · New York

E-ISSN 2196-9736

\section{Corresponding author}

Shyam Menon

Department of

Gastroenterology

The Royal Wolverhampton

Hospitals NHS Trust

New Cross Hospital

Wolverhampton WV6 8AY

United Kingdom

shyam.menon@nhs.net

dr.s.menon@gmail.com
License terms

(이요 $\Theta \circledast$
Background and study aims: Upper gastrointestinal (UGI) cancer in the Western world usually presents at an advanced stage, when opportunities for curative therapy are limited. The failure to detect subtle, early-stage UGI cancer at endoscopy may contribute to a poor prognosis. We undertook a meta-analysis of studies of endoscopic miss rates for UGI cancer to quantify how often opportunities to diagnose cancer at an earlier stage are missed.

Patients and methods: A MEDLINE search was conducted to identify relevant studies, and a meta-analysis was conducted. "Missed" UGI cancer was defined as cancer that had not been diagnosed by UGI endoscopy performed within 3 years before the diagnosis. Random effects metaanalysis was used to determine the event rate of missed UGI cancer.

Results: Ten studies were identified that included 3,787 patients with UGI cancer. Four hundred

\section{Introduction}

\section{$\nabla$}

Upper gastrointestinal (UGI) cancers (of the oesophagus and stomach) characteristically present at a late stage in the Western world. This contributes to the poor overall 5-year survival rates of patients with UGI cancer, which are $13 \%$ for those with oesophageal cancer and $17 \%$ for those with gastric cancer [1]. However, when UGI cancers are diagnosed at an early stage, 5-year survival rates can be as high as $80 \%$ [2].

Patients who undergo a colonoscopy procedure that does not diagnose colorectal cancer are at a low risk of between $0.5 \%$ and $6 \%$ for the subsequent development of colorectal cancer within 3 years $[2,3]$. Whereas awareness of the importance of adenomatous polyps to the subsequent development of colorectal cancer is universal, awareness of the endoscopic appearance of premalignant lesions and early cancers in the oesophagus and stomach appears to be much eighty-seven UGI cancers were missed at endoscopy within 3 years before diagnosis. Marked heterogeneity was observed between studies $\left(I^{2}\right.$, $94.4 \% ; P<0.001)$. On random effects meta-analysis, the pooled miss rates were $6.4 \%$ (95\% confidence interval $[\mathrm{CI}], 4.3 \%-9.5 \%)$ within 1 year and $11.3 \%(95 \% \mathrm{CI}, 7.5 \%-16.6 \%)$ within 3 years before diagnosis. There appeared to be no difference between the miss rates of oesophageal (44\%) and gastric $(51 \%)$ cancer $(P=0.42)$.

Conclusion It appears that $11.3 \%$ of UGI cancers are missed at endoscopy up to 3 years before diagnosis. To ameliorate the poor prognosis of patients with UGI cancer in the Western world, efforts should be made to improve the quality of UGI endoscopy and create opportunities for earlier diagnosis.

less widespread among endoscopists in the Western world. It would be expected, therefore, that patients are undergoing UGI endoscopic procedures that fail to diagnose UGI cancers in the years before the UGI cancers are finally diagnosed, and that crucial opportunities to diagnose these cancers at an earlier and more treatable stage are being missed.

We have undertaken a meta-analysis of studies of how frequently UGI endoscopy fails to diagnose cancer in patients in whom UGI cancer is subsequently diagnosed, so as to quantify how commonly these missed diagnoses occur and examine potential risk factors for missed diagnoses.

\section{Methods}

MEDLINE (U.S.National Library of Medicine, 1966 - 2009) was searched to extract all studies that included the key words missed cancer, missed 


\begin{tabular}{|lllll|}
\hline First author & Year published & Study period & Region & $\begin{array}{l}\text { Sample size } \\
\text { (total number of cancers) }\end{array}$ \\
\hline Hosokawa [4] & 1998 & $1984-1989$ & Japan & 770 \\
\hline Amin [5] & 2002 & $1994-1999$ & UK & 129 \\
\hline Suvakovic [6] & 1997 & $1989-1994$ & UK & 81 \\
\hline Yalamarthi [7] & 2004 & $1996-2001$ & UK & 305 \\
\hline Voutilainen [8] & 2005 & $1994-2001$ & Finland & 284 \\
\hline Hosokawa [11] & 2007 & $1990-1995$ & Japan & 730 \\
\hline Raftopoulos [9] & 2010 & $1990-2004$ & Australia & 822 \\
\hline Milestone [12] & 2007 & $2000-2006$ & UK & 248 \\
\hline Vradelis [10] & 2011 & $2005-2008$ & UK & 74 \\
\hline Vesey [13] & 2013 & $2002-2009$ & UK & 344 \\
\hline
\end{tabular}

Table 1 Summary of studies included in the meta-analysis. upper gastrointestinal cancer, missed oesophageal cancer, and missed gastric cancer. Only studies of adults (age older than 18 years) and studies in English were included. Studies were included in the meta-analysis if they investigated retrospectively either of the following: (1) whether patients with UGI cancer had undergone an endoscopic procedure before the diagnosis that failed to diagnose the cancer or (2) whether patients who had undergone endoscopy without being given a diagnosis of UGI cancer were subsequently given a diagnosis of UGI cancer.

Abstracts were reviewed, and studies that met the above criteria were examined in detail. Reference lists were hand-searched for additional studies. Abstracts presented at national and international meetings (British Society of Gastroenterology, Digestive Disease Week, United European Gastroenterology Week) in the past 5 years were also searched. Studies were ultimately included in the meta-analysis based on agreement by the two investigators.

Missed cancers were defined as cancers in subjects who had undergone an endoscopic procedure that did not diagnose UGI cancer in the 3 years before they were given a diagnosis of UGI cancer.

Random effects meta-analysis was performed to obtain a pooled endoscopic missed UGI cancer rate, and $I^{2}$ values were computed to assess heterogeneity. Comprehensive Meta-Analysis Version 2, Biostat, Englewood, New Jersey (2005), was used for analysis. Forest plots were created to allow studies and their 95\% confidence intervals to be compared. Publication bias was assessed with a funnel plot and the classic fail safe $\mathrm{N}$ test.

\section{Results}

The MEDLINE search yielded 22 studies, 8 of which met the inclusion criteria [4-11]. Two abstracts that fulfilled the inclusion criteria were also included [12,13]. These 10 studies included 3,787 subjects, among whom 487 (12.9\%) had undergone an endoscopic procedure that missed UGI cancer in the 3 years before diagnosis ( Table 1 ). Of these UGI cancers, 49 (10.1\%) were oesophageal cancers and 414 (85\%) were gastric cancers ( $\square$ Table 2). Four duodenal cancers were also included in one study [9], and in another study, published as an abstract, 20 UGI cancers were not subdivided into oesophageal and gastric cancers [13]. Six of the studies examined only the rate of missed gastric cancer at endoscopy [4,7-11].

Data on the total number of endoscopic procedures performed during the study period were available for eight studies $[4,6,9$, 11-13]. A total of 181,662 procedures in these eight studies were associated with 456 missed cancers, with an overall UGI cancer prevalence of $2.1 \%$ and a missed cancer prevalence of $0.25 \%$. In these studies, UGI cancer was diagnosed at 1 in every 48 endoscopies, and UGI cancer was missed at 1 in every 398 endoscopies.

The mean age of subjects with UGI cancer missed at endoscopy in three studies was 70 (range, 66-72) years [7-9]. Missed cancer appeared more common in male (79\%) than in female (21\%) patients in one study, but this difference was not statistically significant (chi-square test, $P=0.29$ ) [9].

\section{Heterogeneity}

Marked heterogeneity was observed between studies: $I^{2}=94.4 \%$, $P<0.001$ for endoscopy missing cancers up to 3 years before diagnosis and $I^{2}=83.2 \%, P<0.001$ for endoscopy missing cancers within 1 year before diagnosis. Therefore, a random effects meta-analysis was performed.

\section{Rate of upper gastrointestinal cancers missed at endoscopy}

Within 3 years before a diagnosis of UGI cancer, 487 subjects had undergone an endoscopic procedure that failed to diagnose their oesophagogastric or duodenal cancer. On random effects meta-analysis, the pooled miss rate of UGI cancer within 3 years before diagnosis was $11.3 \%$ (7.5\% - 16.6\%; • Fig. 1).

One hundred forty-three (29\%) subjects had undergone an endoscopic procedure that failed to diagnose their cancers within 1 year before diagnosis. Of these, 33 (23\%) were oesophageal cancers, 96 (67\%) were gastric cancers, and 3 (2\%) were duodenal cancers. No data were available on the site of cancer in $11(8 \%)$. On random effects meta-analysis, the pooled miss rate of UGI cancer within 1 year before diagnosis was 6.4\% (4.3\%-9.5\%; - Fig. 2).

Three hundred forty-four (71\%) subjects had undergone an endoscopic procedure that failed to diagnose their cancers between 1 and 3 years before diagnosis. Of these, 31 (9\%) were oesophageal cancers, 303 (88\%) were gastric cancers, and $1(0.2 \%)$ was a duodenal cancer. No data were available on the site of cancer in 9 cases (2.6\%). On random effects meta-analysis, the pooled miss rate of UGI cancer between 1 and 3 years before diagnosis was $6.3 \%(3.1 \%-12.4 \%)$.

\section{Site of Cancer}

Of the 10 studies examined in this meta-analysis, 6 studies did not include oesophageal cancers. In the 3 studies that included both oesophageal and gastric cancers, $51 \%$ of the missed cancers were gastric cancers and $44 \%$ were oesophageal cancers (chi- 
Table 2 Summary of the total number of upper gastrointestinal cancers and subjects undergoing endoscopy in the previous 3 years that failed to diagnose the cancer in each study.

\begin{tabular}{|c|c|c|c|c|c|c|}
\hline First author & $\begin{array}{l}\text { Missed cancers } \\
\text { (OGD within } 3 \\
\text { years before } \\
\text { diagnosis) }\end{array}$ & $\begin{array}{l}\text { Oesophageal can- } \\
\text { cers }\end{array}$ & Gastric cancers & Duodenal cancers & Total cancers & $\begin{array}{l}\text { Prevalence of } \\
\text { missed cancers (\%) }\end{array}$ \\
\hline Hosokawa [4] & 111 & NA & 111 & NA & 770 & 14.4 \\
\hline Amin [5] & 18 & NA & 18 & NA & 129 & 14.0 \\
\hline Suvakovic [6] & 11 & NA & 11 & NA & 81 & 13.6 \\
\hline Yalamarthi [7] & 30 & 16 & 14 & NA & 305 & 9.8 \\
\hline Voutilainen [8] & 13 & NA & 13 & NA & 284 & 4.6 \\
\hline Hosokawa [11] & 188 & NA & 188 & NA & 730 & 25.8 \\
\hline Raftopoulos [9] & 55 & 16 & 35 & 4 & 822 & 6.7 \\
\hline Milestone [12] & 25 & 17 & 8 & NA & 248 & 10.1 \\
\hline Vradelis [10] & 16 & NA & 16 & NA & 74 & 21.6 \\
\hline Vesey [13] & 20 & Not provided & Not provided & NA & 344 & 5.8 \\
\hline
\end{tabular}

Abbreviation: OGD, oesophagogastroduodenoscopy.

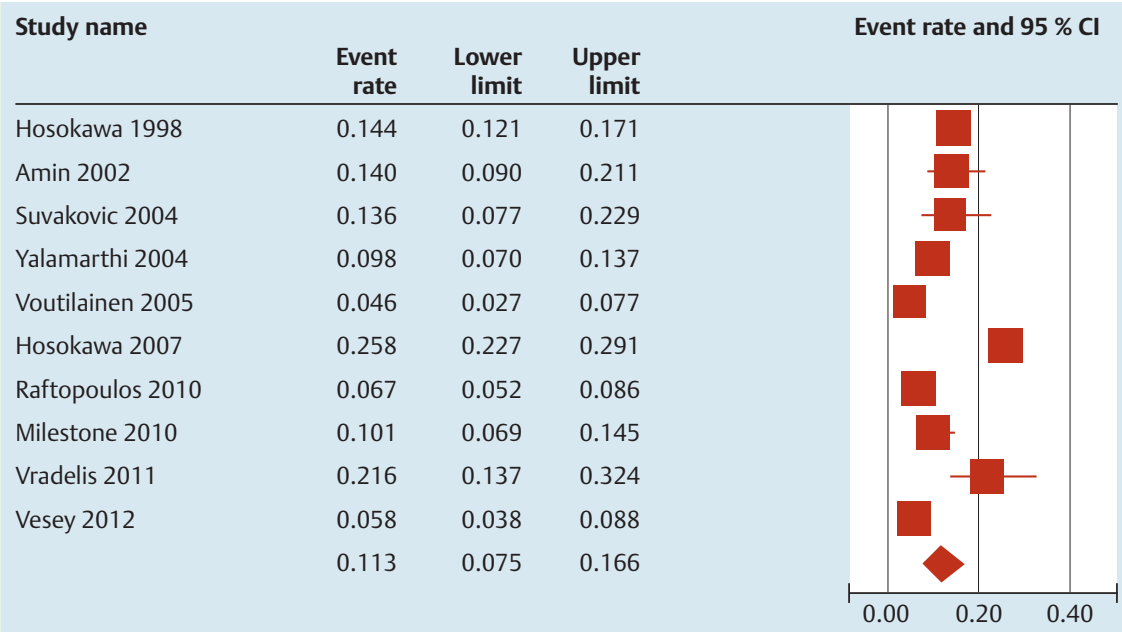

Fig. 1 Forest plot of subjects undergoing endoscopy that failed to diagnose upper gastrointestinal cancer within 3 years before the diagnosis.

\begin{tabular}{lrrr} 
Study name & $\begin{array}{r}\text { Event } \\
\text { rate }\end{array}$ & $\begin{array}{r}\text { Lower } \\
\text { limit }\end{array}$ & $\begin{array}{r}\text { Upper } \\
\text { limit }\end{array}$ \\
\hline Hosokawa 1998 & 0.049 & 0.036 & 0.067 \\
Amin 2002 & 0.140 & 0.090 & 0.211 \\
Yalamarthi 2004 & 0.066 & 0.043 & 0.099 \\
Raftopoulos 2010 & 0.035 & 0.025 & 0.050 \\
Milestone 2010 & 0.069 & 0.043 & 0.107 \\
Vradelis 2011 & 0.135 & 0.074 & 0.233 \\
Vesey 2012 & 0.032 & 0.018 & 0.057 \\
& 0.064 & 0.043 & 0.095
\end{tabular}

square test, $p=0.42)[7,9,12]$. One study did not provide data on the breakdown of oesophageal and gastric cancers [13].

\section{Publication bias}

Publication bias was examined by creating a funnel plot of the log transformed event rates for the individual studies ( $\bullet$ Fig. 3 ). The plot shows that the majority of the studies are scattered around the point estimate. The classic fail safe $\mathrm{N}$ test suggests an $\mathrm{N}$ value of 3,190 (for $z=-35.1$, two-tailed $P<0.001$ ), which makes publication bias unlikely because 3,190 "null" studies would be needed for the combined two-tailed $P$-value to exceed 0.05 , or 319
Event rate and $95 \% \mathrm{Cl}$ Fig. 2 Forest plot of subjects undergoing endoscopy that failed to diagnose upper gastrointestinal cancer within 1 year before the diagnosis. 


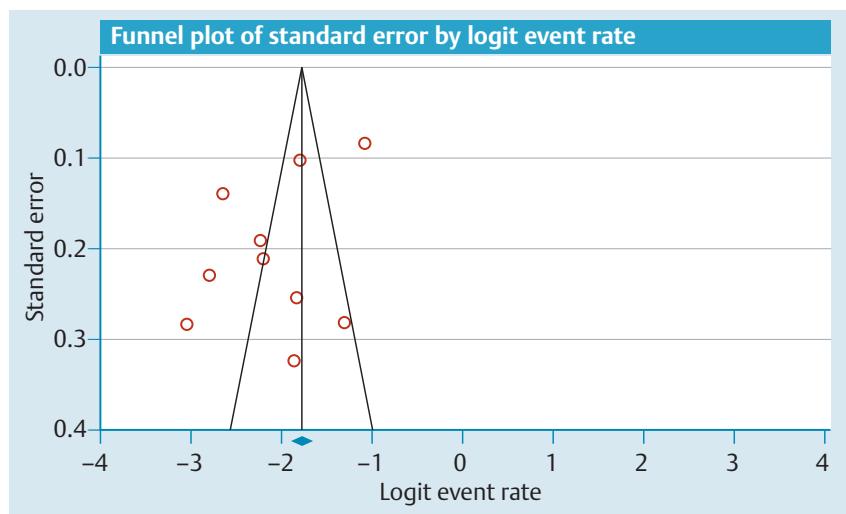

Fig. 3 Funnel plot of studies of the rate of endoscopy failing to diagnose upper gastrointestinal cancer to assess for publication bias.

a macroscopic lesion at the time of the initial endoscopy and therefore had been missed. If one assumes that the doubling time for mucosal cancers is 2 to 3 years, UGI cancers diagnosed within 2 to 3 years after a normal endoscopy may well have been associated with a mucosal lesion at the time of endoscopy and are therefore regarded as "possibly missed."

In this meta-analysis, failure to diagnose or missing a UGI cancer at endoscopy was a relatively common event among patients with UGI cancer, occurring in $6.4 \%$ within 1 year before diagnosis and $11.3 \%$ up to 3 years before diagnosis. However, it is important to recognize that this is a relatively uncommon event in all patients undergoing endoscopy, occurring in 1 in every 398 endoscopic procedures.

Two series from the same unit in Japan reported high rates (14\% and $26 \%$ ) of missed gastric cancer, diagnosed within 3 years after endoscopy that did not diagnose cancer $[4,11]$. Male subjects and endoscopists with fewer than 10 years of experience were associated with failure to detect gastric cancer at endoscopy. Neither series included oesophageal cancer in its analysis.

A Scottish group subsequently reviewed 305 subjects (among 13,589 patients undergoing endoscopy) with oesophageal and gastric cancer and found that 30 had undergone a minimum of one endoscopic procedure during the preceding 3 years [7]. Surprisingly, $75 \%$ of the patients with missed oesophageal cancers and $59 \%$ of those with missed gastric cancers had had alarm symptoms of dysphagia, anemia, haematemesis, weight loss, or vomiting at the time of the endoscopy that missed the cancer. Detailed analysis of individual cases revealed that $27 \%$ of the missed UGI cancer cases were due to pathologist error and the remaining $73 \%$ due a variety of endoscopist errors, including not detecting a lesion, detecting an abnormality but not taking a biopsy, detecting an abnormality for which the biopsy was benign, taking an insufficient biopsy, and inappropriate delays in follow-up. The same group recently reported their experience of concentrating UGI endoscopy in the hands of two experienced individuals [13]. Between 2002 and 2009, these two endoscopists performed 16,503 procedures, and there was a reduction from $7 \%$ to $3.2 \%$ in the percentage of UGI cancers missed at endoscopy within 1 year before diagnosis. However, this reduction appeared to be due to a reduction in pathology and follow-up errors, rather than improvement in endoscopy performance.

More than 28,000 endoscopic procedures were examined at a single institution in Western Australia, and of the 822 UGI cancers, 29 were missed cancers (endoscopy did not diagnose cancer within 1 year before diagnosis) and 26 were possible missed can- cers (endoscopy did not diagnose cancer between 1 and 3 years before diagnosis) [9]. Again, alarm symptoms were associated with an increased risk for missing cancer at endoscopy. In 69\% of the missed UGI cancer cases, an abnormality was described at initial endoscopy that was at the same site as the subsequent malignancy. The authors also noted that squamous cell carcinoma in the proximal esophagus seemed to be a commonly missed lesion in their series.

In a small series of 74 patients with a diagnosis of gastric cancer in Oxford in the United Kingdom, taking fewer biopsy specimens at endoscopy was found to be associated with an increased risk for missing UGI cancer [10]. Milestone et al reviewed data on oesophageal and gastric cancers from 2000 to 2006 at a single center in the United Kingdom and reported that 25 of 248 patients had undergone an endoscopic procedure within 3 years before diagnosis that failed to diagnose their cancer [12]. Forty-seven percent of these missed lesions were in the same position as previously documented endoscopic abnormalities.

There are several possible explanations for a "missed" upper GI cancer. These include technical limitations in endoscopy technique and lesion recognition; inadequate supervision of trainees; sampling error (too few or inaccurate biopsies); lack of patient tolerance of the procedure or inadequate sedation, resulting in a poor or incomplete mucosal assessment; inappropriate followup; and errors of histopathology interpretation. The majority of the missed cancers included in this meta-analysis were gastric cancers. The large surface area of the stomach may present particular challenges to complete examination. The Japanese experience of gastric cancer emphasizes the importance of meticulous UGI endoscopy. This entails preparation of the patient with a defoaming agent combined with a mucolytic agent to improve visibility; careful, systematic inspection of the stomach with adequate air insufflation to flatten the gastric folds; and extensive photographic documentation (>25 images) to ensure adequate views of all areas of the stomach [15]. Clinical trials of defoaming and mucolytic agents have shown that the administration of such agents does improve mucosal visualization [16].

In the studies that reported endoscopic findings, at least half of the patients with a missed UGI cancer had an abnormality described at the site of the cancer that was either not biopsied or biopsied insufficiently $[9,12]$. A greater number of biopsies taken at endoscopy correlates with the likelihood of a "positive" diagnosis. Increasing the number of biopsies from two to six improved the diagnostic yield for oesophageal cancer from 95.8 to $100 \%$ [17]. An accuracy rate of up to $97 \%$ for diagnosing gastric cancer was achieved when at least five biopsy samples were taken [18].

The marked heterogeneity among the studies examined in this meta-analysis raises issues about the justification of a meta-analytic approach. This is a particularly pertinent issue when the definition of the clinical variable being examined is inconsistent and the methodology of the studies varies, as is the case in a systematic review. However, the studies examined in this meta-analysis have a uniform methodology and a clear definition of "missed" cancer, and we believe that a meta-analytic approach to these studies is therefore justified. Furthermore, given the degree of heterogeneity among the studies, we feel that a random effects model was justified [19].

There are several limitations to our meta-analysis. The diagnosis of "missed" UGI cancer was based on a retrospective review of the data, and although similar methodologies were employed in the studies examined, not surprisingly, significantly heteroge- 
neous results were found given the variety of countries, variable endoscopic practices, and differing incidence rates of UGI cancer. It was therefore not possible to characterize further the various causes of missing UGI cancer at endoscopy, whether related to the procedure, the organization of follow-up, or histopathology. One of the studies included used a shorter time frame of 2 rather than 3 years before diagnosis to define a case of missed UGI cancer, which may have slightly underestimated the number of missed UGI cancers in this population [5]. Studies were not explicit in defining an appropriate follow-up interval, with the exception of a study from Oxford that very generously allowed up to 6 months to be an appropriate follow-up interval after an endoscopy that failed to diagnose cancer [10]. The majority of subjects included in the meta-analysis had gastric cancer. Oesophageal cancer appeared just as likely to be missed as gastric cancer, but this conclusion is based on far fewer data. Additionally, data on age and sex were available from only three studies and one study, respectively, thus limiting the interpretation of the influence of age and sex on the prevalence of missed cancers.

In summary, $11.3 \%$ of UGI cancers were missed at endoscopy during the 3 years before diagnosis. Individual studies suggest that male gender, presentation with alarm symptoms, endoscopists with less experience, pathology errors, failure to biopsy lesions adequately, follow-up errors, and squamous cell carcinoma of the esophagus may all be important factors in the failure to detect cancer at UGI endoscopy. Given the impact that an endoscopic procedure that fails to diagnose cancer often has on patients, their relatives, and their clinicians, studies to improve the quality of UGI endoscopy in the Western world and continuing efforts to minimise histopathologic and follow-up errors are needed.

\section{Competing interests: None}

\section{References}

1 Cancer Research UK. http://www.cancerresearchuk.org/cancer-info/ cancerstats/types/oesophagus/survival/ and http://www.cancerresearchuk.org/cancer-info/cancerstats/types/stomach/ Accessed March 4, 2-13

2 Bressler B, Paszat LF, Vinden C et al. Colonoscopic miss rates for rightsided colon cancer: a population-based analysis. Gastroenterology 2004; $127: 452-456$
3 Bressler B, Paszat LF, Chen Z et al. Rates of new or missed colorectal cancers after colonoscopy and their risk factors: a population-based analysis. Gastroenterology 2007; 132: $96-102$

4 Hosokawa O, Tsuda S, Kidani E et al. Diagnosis of gastric cancer up to three years after negative upper gastrointestinal endoscopy. Endoscopy 1998; 30: 669-674

5 Amin A, Gilmour H, Graham L et al. Gastric adenocarcinoma missed at endoscopy. J R Coll Surg Edinb 2002; 47: 681-684

6 Suvakovic $Z$, Bramble MG, Jones $R$ et al. Improving the detection rate of early gastric cancer requires more than open access gastroscopy: a five year study. Gut 1997; 41: $308-313$

7 Yalamarthi S, Witherspoon P, McCole D et al. Missed diagnoses in patients with upper gastrointestinal cancers. Endoscopy 2004; 36: $874-879$

8 Voutilainen ME, Juhola MT. Evaluation of the diagnostic accuracy of gastroscopy to detect gastric tumours: clinicopathological features and prognosis of patients with gastric cancer missed on endoscopy. Eur J Gastroenterol Hepatol 2005; 17: 1345-1349

9 Raftopoulos SC, Segarajasingam DS, Burke Vet al. A cohort study of missed and new cancers after esophagogastroduodenoscopy. Am J Gastroenterol 2010; 105: $1292-1297$

10 Vradelis S, Maynard N, Warren BF et al. Quality control in upper gastrointestinal endoscopy: detection rates of gastric cancer in Oxford 20052008. Postgrad Med J 2011; 87: 335 -339

11 Hosokawa O, Hattori M, Douden K et al. Difference in accuracy between gastroscopy and colonoscopy for detection of cancer. Hepatogastroenterology 2007; 54: $442-444$

12 Milestone AN, Kent AJ, Goldin R et al. Missed upper gastrointestinal cancer at endoscopy. Gastroenterology 2007; 132: A-312 S2080

13 Vesey AT, Auld CD, McCole D. Missed upper gastrointestinal cancer at endoscopy: can performance be improved by specialists? Gut 2012; 61: A151-A152

14 Fujita S. Biology of early gastric carcinoma. Pathol Res Pract 1978; 163 : 297-309

15 Ang TL, Khor CJ, Gotoda T. Diagnosis and endoscopic resection of early gastric cancer. Singapore Med J 2010; 51: $93-100$

$16 \mathrm{Kuo} \mathrm{CH}$, Sheu BS, Kao AW et al. A defoaming agent should be used with pronase premedication to improve visibility in upper gastrointestinal endoscopy. Endoscopy 2002; 34: $531-534$

17 Lal N, Bhasin DK, Malik AK et al. Optimal number of biopsy specimens in the diagnosis of carcinoma of the oesophagus. Gut 1992; 33: $724-$ 726

18 Tatsuta M, Iishi H, Okuda S et al. Prospective evaluation of diagnostic accuracy of gastrofiberscopic biopsy in diagnosis of gastric cancer. Cancer 1989; 63: 1415-1420

19 Kontopantelis E, Springate DA, Reeves D. A re-analysis of the Cochrane Library data: the dangers of unobserved heterogeneity in meta-analyses. PLoS ONE 2013; 8: e69930 DOI 10.1371/journal.pone.0069930 